\title{
Introduction to the special issue on quantified CSPs and QBF
}

\author{
Enrico Giunchglia • Kostas Stergiou
}

Published online: 13 July 2008

(C) Springer Science + Business Media, LLC 2008

Constraint satisfaction problems (CSPs) and satisfiability problem (SAT) are very successful frameworks that are used to model and solve a wide variety of combinatorial problems. However, there are classes of problems containing uncertainty that arise in areas such as contingent planning, adversarial game playing, control design, and model checking that cannot be expressed within these frameworks. Typically, such problems involve decisions or events that are beyond the control of the problem solving agent and thus cannot be handled using standard (existentially quantified) variables. Quantified CSPs and quantified Boolean formulae (QBF), which are the extensions of CSPs and SAT that allow for universally quantified variables, make it possible to model and reason with such problems, as well as other problems that contain "bounded uncertainty". As a result, these frameworks have been attracting significant interest in recent years. The main advances have been achieved in the area of QBF where numerous solvers have been implemented and real problems of considerable size have been tackled. There is also a significant body of work on quantified numerical constraints over continuous domains, while research works on quantified constraint satisfaction problems (QCSPs) with discrete finite domains have started to emerge. With this special issue of Constraints we aim to group together and reflect the state of the art in these rapidly developing areas of research.

The papers in this special issue concern the three areas of reasoning with quantified constraints highlighted above.

E. Giunchglia $(\bowtie)$

Universita di Genova, Genoa, Italy

e-mail: enrico@dist.unige.it

K. Stergiou

University of the Aegean, Mytilene, Greece

e-mail: konsterg@aegean.gr 
QCSPs with discrete finite domains Bodirsky and Chen study the relatively quantified constraint satisfaction problem (RQCSP), a subclass of the QCSP in which the values for each individual variable can be arbitrarily restricted. They give a complete complexity classification of the cases of the RQCSP where the types of constraints that may appear are specified by a constraint language.

Stynes and Brown propose two families of value ordering heuristics for QCSPs. The first is inspired by adversarial search while the second attempts to reduce the size of the universal variables' domains taking advantage of the pure value rule.

Quantified Boolean formula Egly, Seidl, and Woltran describe a QBF solver, called "qpro", which can handle QBFs expressed not only in prenex conjunctive normal form, but in any arbitrary way. They extend algorithms for QBFs to the nonnormal form case and compare qpro with the leading normal-form solvers on several problems from the area of artificial intelligence.

Pulina and Tacchella study the problem of engineering a robust solver for QBF. Using inductive reasoning techniques to learn engine-selection policies they obtain a multiengine solver, i.e., a tool which can choose among a number of state-of-the-art QBF solvers the one which is more likely to yield optimal results. They also improve the multi-engine solver by adding the capability of updating the learned policies when they fail to give good predictions. In this way the solver becomes also self-adaptive.

QCSPs with continuous domains Goldsztejn, Michel, and Rueher present a new algorithm for solving quantified CSPs defined by universally quantified inequalities on continuous domains. They introduce a generic branch and prune algorithm to tackle continuous CSPs, where pruning and solution identification are dedicated for universally quantified inequalities. Special rules are proposed to handle the parameters domains of the constraints.

We would like to thank all the reviewers for contributing their time and expertise to this special issue. We are also grateful to Peter van Beek, Editor-in-chief of Constraints, who invited us to edit this special issue. 